\title{
Impact of haematopoietic stem cell transplantation with total body irradiation on apparent bone mineral density in childhood leukaemia survivors
} C Wei ${ }^{1,2,3}$, R Cox ${ }^{4}$, KJ Bradley2,5, R Elson ${ }^{1,4}$, MCG Stevens ${ }^{2,4}$, EC Crowne ${ }^{1,2}$

1. Department of Paediatric Endocrinology \& Diabetes, Bristol Royal Hospital for Children (BRHC), U.K.; 2. University of Bristol, U.K.; 3. Department of Paediatric Endocrinology \& Diabetes, St Georges Hospital, London, UK

4. Department of Paediatric Oncology, BRHC, U.K.; 5. Department of Endocrinology and Diabetes, Bristol Royal Infirmary, U.K.

\section{Background}

- Childhood HSCT/TBI survivors have multiple risk factors for reduced bone mineral density (BMD) and poor growth.

- Reduced Z-scores from Dual energy X-Ray absorptiometry (DEXA) have been reported.

- However, smaller bones have apparently lower areal BMD than larger ones (figure 1). DEXA measurements may be difficult to interpret in childhood HSCT/TBI survivors who are often short.

- Bone mineral apparent density (BMAD) allows correction of BMD for size in patients with short stature.

Figure 1: DEXA BMD does not take into account of the depth of the bone. The smaller bone has an apparently lower areal BMD than the larger one despite having the same volumetric density (Carter et al).
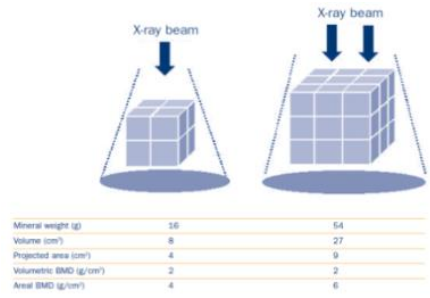

\section{Aim}

To investigate the influence of HSCT/TBI on size-corrected $\mathrm{BMD}$ in childhood leukaemia survivors

\section{Method}

Participants 2 Groups (all post-pubertal):

- Group 1: BMT/TBI ALL survivors diagnosed at <18 yrs, in remission $\geq 3$ yrs

- Group 2: Standard chemotherapy treated ALL survivors diagnosed at $<18 \mathrm{yrs}$ in remission $\geq 3 \mathrm{yrs}$

\section{Exclusion:}

- Currently on steroids, untreated endocrinopathies, pregnancy

\section{Assessments \& definitions}

- Auxology: height, weight

- DEXA scanning(Lunar Prodigy® fan beam): BMD-Z-scores, bone mineral content (BMC), bone area (BA), bone width

- Vitamin D levels

Definition of size-corrected BMD using BMAD:

- Total-BMAD $\left(\mathrm{BMAD}_{\mathrm{T}}\right)=\mathrm{BMC} /$ total body $B \mathrm{~A}^{\wedge} 2 /$ height (Ref: Katzman et al.J Clin Endocrinol Metab 1991:73:1332-1339)

- Lumbar spine-BMAD $\left(\mathrm{BMAD}_{\mathrm{L2}-4}\right)=\mathrm{BMD}_{\mathrm{L} 2-4} \times[4 /(\pi \mathrm{x}$ width $)]$ (Ref: Carter et al. Bone Miner Res. 1992; 7: 137-145.)

Analysis: Student's t-tests, Pearson's correlations

Approval: Regional Research Ethics Committee

\section{Results}

\section{Table 1: Demographics}

\begin{tabular}{|c|c|c|}
\hline & $\begin{array}{l}\text { HSCT/TBI } \\
(n=21,11 M)\end{array}$ & $\begin{array}{l}\text { Chemo-only } \\
(n=31,13 M)\end{array}$ \\
\hline Current age (years) ${ }^{*}$ & $21.5(16.2-26)$ & $21.5(16.2-26)$ \\
\hline Age at primary diagnosis (years) * & $5.3(1.0-10.8)$ & $7.0(1.6-18.0)$ \\
\hline Age of HSCT/TBI (years)* & $9.3(2.6-16.7)$ & NA \\
\hline $\begin{array}{l}\text { Endocrinopathies: } \\
\text { Growth hormone Deficiency } \\
\text { Hypothyroidism } \\
\text { Hypogonadism }\end{array}$ & $\begin{array}{l}12 \\
10 \\
17\end{array}$ & $\begin{array}{l}0 \\
0 \\
0\end{array}$ \\
\hline
\end{tabular}

Table 2. Baseline measurements: HSCT/TBI survivors compared with are shorter with lower lean mass than chemotherapy-only survivors

\begin{tabular}{|l|c|c|c|}
\hline $\begin{array}{c}\text { Group(s) } \\
\text { Variable }\end{array}$ & $\begin{array}{c}\text { HSCT/TBI } \\
(\mathbf{n}=21)\end{array}$ & $\begin{array}{c}\text { Chemo-only } \\
(\mathbf{n}=31)\end{array}$ & $\begin{array}{c}\text { t-test } \\
\mathbf{p}\end{array}$ \\
\hline Height SDS & $-1.4(1.5)$ & $0.2(0.9)$ & $<0.001$ \\
\hline Weight SDS & $-1.6(2.5)$ & $1.1(1.3)$ & $<0.001$ \\
\hline Fat Mass $(\mathrm{kg})$ & $15.1(10.1)$ & $21.6(10.4)$ & 0.06 \\
\hline Lean mass $(\mathrm{kg})$ & $36.9(10.2)$ & $45.0(10.8)$ & 0.009 \\
\hline
\end{tabular}

Figure 2. HSCT/TBI survivors had lower total-BMD Z scores

However, size corrected is needed for interpretation as total-BMD correlated positively with heightSDS, weight-SDS, fat and lean masses (all $p \leq 0.001$ ).

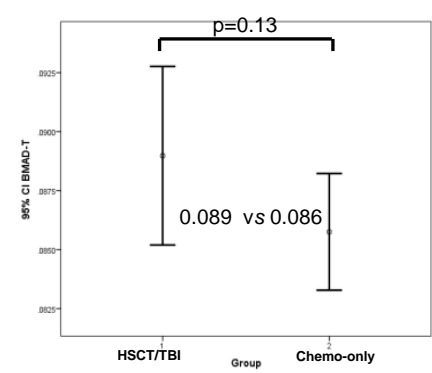

Figure 3. Total BMAD-Z scores
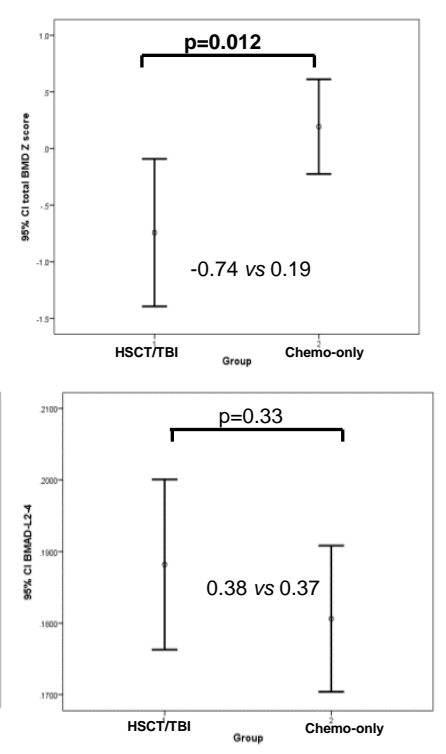

Figure 4. Lumbar spine BMAD Z scores
There were no mean(SD) differences in size-corrected BMD between HSCT and chemotherapy-only patients (Figures 3 and 4).

Table 3. BMAD was not associated with treatment factors, vitamin D levels or endocrinopathies in HSCT survivors identified

\begin{tabular}{|l|c|c|}
\hline & $\begin{array}{c}\text { BMAD }_{\mathrm{T}} \\
\text { p-value }\end{array}$ & $\begin{array}{c}\text { BMAD }_{\text {L2-4 }} \\
\text { p-value }\end{array}$ \\
\hline Age of primary treatment & 0.36 & 0.67 \\
\hline Age of HSCT/TBI & 0.23 & 0.68 \\
\hline HSCT before or after 8 years & 0.35 & 0.75 \\
\hline Vitamin D levels & 0.13 & 0.21 \\
\hline $\begin{array}{l}\text { Endocrine disorders: } \\
\text { Growth hormone deficiency }\end{array}$ & & \\
\hline $\begin{array}{l}\text { Hypothyroidism } \\
\text { Gonadal failure }\end{array}$ & 0.16 & 0.46 \\
\hline
\end{tabular}

\section{Summary and Conclusions}

- Size of patient must be taken into account to avoid over diagnosis of osteopenia when assessing BMD in cancer survivors.

- Treatment effects on peak bone mass in survivors need further evaluation.

Acknowledgements: The study was funded by the IPSEN clinical research fellowship, BSPED research Award, David Telling Research funds, Peel Medical research award, NIHR feasibility \& sustainability funds. 